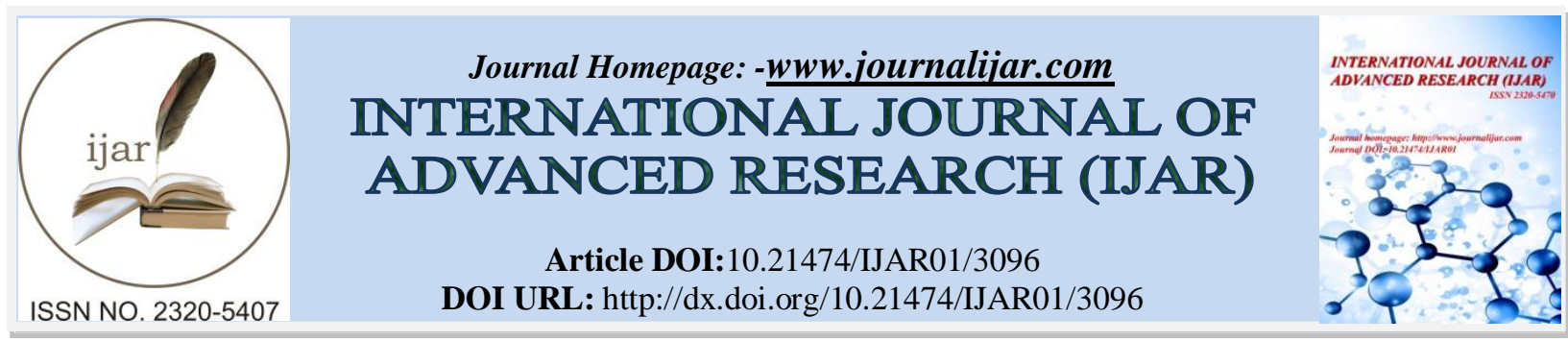

RESEARCH ARTICLE

\title{
EXPLORING PREVALENCE OF LONG TERM DIABETES MELLITUS VASCULAR COMPLICATIONS IN KING FAHAD GENERAL HOSPITAL
}

\section{Wyyed Al Harbi, Daniah Ahmad Faraj, Lubna Ismail, Lujain Asiri and Alia Ismail. King Fahad General Hospital.}

\section{Manuscript Info}

Manuscript History

Received: 03 November 2016

Final Accepted: 28 December 2016

Published: January 2017

\section{Abstract}

Study objective(s):-

Primary objective:-

To assess the cumulative and individual prevalence of microvascular and macrovascular complications among study population.

Secondary objective(s)

- To Map Cardiovascular Risk Factors among study population.

- Correlate diabetic complication prevalence with level of diabetes control (A1C target level)

Study Design \&Duration:-

A retrospective cross sectional study design will be used. Patients' files will be used as source of data collection in a brief case report form. To avoid duplication unique patient file number will be used as subject number preceded by investigator number. A random data source verification of $20 \%$ of the case report forms as quality control of collected data. Patients will be enrolled inn 1 month

\section{Study population:-}

Inclusion criteria

- Male or Female patients of all age groups.

- Established diagnosis of DM for more than 10 years.

- Patients presented to the outpatient clinic during the last three months.

Exclusion criteria

- Incomplete patient's file

Expected number of patients: 375 patients attending to King Fahad General Hospital, Jeddah, Saudi Arabia.

Expected number of investigators: 3 investigators.

Recruitment modalities:-

Selection of patients

In order to allow extrapolation of the results to the broadest possible population, all consecutive patients who meet the eligibility criteria and agree to participate upon a signed consent form will be included in the study. Each physician should recruit about 125 consecutive DM patients. 


\section{Endpoint(s):-}

Primary endpoints

- Number of Micro/Macrovascular complications

- Proportion of patients having micro/macro complications

- Frequency of micro/macrovascular complications per patients

- Proportion of individual micro/macrovascular complication among study population.

Secondary endpoints

- Proportion of Cardiovascular risk factors among study population and its correlation to DM vascular complications

Difference in prevalence of micro/macrovascular and individual complications in patients with target $\mathrm{A1C}<8 \%$ or $>8 \%$ at the time of diagnosis MAIN DATA COLLECTED:-

Data will be collected during a single visit in an individual case report form (CRF). No tests will be specifically done for this study.

The following data will be collected:

- Date of last visit,

- Inclusion/exclusion criteria check,

- Patient profile:

- Demographic characteristics (age, gender, marital status, etc.),

- Lifestyle habits (smoking status, physical activity),

- Vital signs, weight, waist circumference, body mass index, blood pressure).

- Family History of DM and Cardiovascular disease

- Relevant history of risk factors for DM

- Characteristics of diabetes:

- $\quad$ Type of Diabetes

- Duration of diabetes,

- Cardiovascular Risk Factors

- Microvascular Complications

- Retinopathy (Date of Diagnosis, A1C level)

- Nephropathy (Date of Diagnosis, A1C level)

- Neuropathy (Date of Diagnosis, A1C level)

- Macrovascular Complications

- Ischemic heart Disease (Type, Date of Diagnosis, A1C level)

- Cerebrovascular Disease (Type, Date of Diagnosis, A1C level)

- Peripheral vascular Disease (Type, Date of Diagnosis, A1C level)

Statistical considerations:-

Sample size calculation

Number of subjects: $\mathrm{N}=375$

Sample size:

Distribution by country or region: Limited to one center as pilot study

Statistical power and sample size justification:-

According to Litwak et al. 2013; ${ }^{14}$ the prevalence of microvascular and macrovascular complications among diabetic patients in Middle East/Gulf area including Saudi Arabia was $65.8 \%$ and $28.7 \%$ respectively; accordingly:

Sample size for Microvascular complications: In our study; it is hypothesized that the prevalence of microvascular complications among diabetic patients would be $60 \%$ with a precision of $\pm 5 \%$. A sample size of 369 individuals will allow for a confidence level of $95 \%$ considering a 2-talied test.

Sample size for Macrovascular complications: In our study; it is hypothesized that the prevalence of macrovascular complications among diabetic patients would be $25 \%$ with a precision of $\pm 5 \%$. A 
sample size of 288 individuals will allow for a confidence level of $95 \%$ considering a 2-talied test.

Statistical Analysis Plan:-

Statistical analysis will be based on all patients enrolled in the registry. Descriptive analysis will be performed. Patient 's characteristics (demographic data, risk profile, treatment, etc.) will be described for all the included patients.

Quantitative data will be summarized using mean, median, standard deviation and range for continuous parameters and counts and percentages for categorical parameters. All statistical tests will be performed using two-tailed tests at a 5\% level of significance. Moreover, statistical test will be performed on comparing different groups. Chi square test will be used to compare between categorical parameters. The results will be calculated at 95\% CI and 5\% level of significance. For parametric data; two-tailed paired t-test and repeated measures ANOVA will be used when comparing between repeated measurements while independent t-test and one way ANOVA when comparing between study groups. Non-parametric tests of significance would be used with data not normally distributed. Logistic Regression analysis would be performed to detect positive and negative predictors of microvascular and macrovascular complications.

Estimated duration of the study:-

Estimated enrollment duration: 1 month

Estimated dates:

- FPI: 1 JAN 2017

- $\quad$ LPI: 31 JAN 2017

- $\quad$ LPO: 31 JAN 2017

- $\quad$ Database lock date: 28 FEB 2017

Report date: 31 MAR 2017

Copy Right, IJAR, 2016,. All rights reserved.

$\begin{array}{ll}\text { List of Abbreviations:- } \\ \text { ADR } & \text { Adverse Drug Reaction } \\ \text { AE } & \text { Adverse Event } \\ \text { CI } & \text { Confidence Interval } \\ \text { CRF } & \text { Case Report Form } \\ \text { CRO } & \text { Contract Research Organization } \\ \text { DM } & \text { Diabetes Mellitus } \\ \text { GCP } & \text { Good Clinical Practice } \\ \text { A1c } & \text { Glycated hemoglobin } \\ \text { ICH } & \text { International Conference on Harmonization } \\ \text { IEC } & \text { Independent Ethics Committee } \\ \text { IRB } & \text { Institutional Review Board } \\ \text { SAP } & \text { Statistical Analysis Plan } \\ \text { SFDA } & \text { Saudi Food and Drug Authority }\end{array}$

\section{Introduction and Rationale:-}

\section{Background:-}

Diabetes is one of the largest global health emergencies of the 21 st century. Each year more and more people live with this condition, which can result in life-changing complications. In addition to the 415 million adults who are estimated to currently have diabetes, there are 318 million adults with impaired glucose tolerance, which puts them at high risk of developing the disease in the future. Nearly each 6 seconds a patient would die because of diabetes and its complications reaching up to 5 million deaths a year ahead of cancer, tuberculosis and Malaria mortalities. ${ }^{1}$ 
The number of people with type 2 diabetes is growing rapidly worldwide. This rise is associated with ageing populations, economic development, increasing urbanization, less healthy diets and reduced physical activity. ${ }^{2}$

Over the past three decades, major social and economic changes have transformed many of the countries in the Middle East and North Africa (MENA) region. Some Gulf States have undergone rapid economic growth and urbanization, associated with reduced infant mortality and increasing life expectancy. ${ }^{3}$

The age adjusted comparative prevalence rate of Diabetes Mellitus across the MENA region is estimated to be $10.7 \%$ with 35.4 million patients suffering from diabetes with 17.1 billion USD health expenditure for Diabetes and costlier 342 thousand deaths a year. The age adjusted prevalence of impaired glucose tolerance account for $8.6 \%$ adding another 30.2 million progressing to overt Diabetes Mellitus. Even Type 1 DM which is the by far less common compared to T2 DM the prevalence is 60.1 Thousand patients. ${ }^{3}$

Saudi Arabia has the highest prevalence across the MENA region in Type II DM reaching $>20 \%$ of adult population aged 20-79 years old. Saudi Arabia also have some of the world's highest annual incidence rates of type 1 diabetes in children, with 31.4 new cases per 100,000 population, hence, Saudi Arabia has 16,100 children with type I diabetes, by far the highest number in the region, and over a quarter of the region's total of $60,700 .{ }^{3,4}$

Moreover, the diabetic population consumes a disproportionate share of health care resources because of both micro-vascular and macro-vascular complications. Strategies to lessen the disease burden in these patients include hygienic measures (diet and exercise) as well as rigorous treatment of hypertension, dyslipidemia and hyperglycemia. $^{5}$

Diabetic patients are at high risks of developing microvascular complications of small blood vessels or macrovascular complications affecting larger blood vessels. ${ }^{6}$ Microvascular complications are long-term complications and can be classified into diabetic retinopathy nephropathy or neuropathy. ${ }^{7}$ The narrowing of arteries leads to decreased blood flow to the heart, or to the brain, or to the limbs leading to several macrovascular complications. $^{8}$

There are a large number of epidemiological studies or national registers which have been conducted worldwide, particularly in the westernized countries, in order to assess the quality of care in diabetic patients, or to check compliance with national treatment guidelines and national program for improvement of quality of care of these patients. There has also been an international collaboration of different registries from various countries aiming to better understand global pattern of the diabetic disease. ${ }^{9,10}$

\section{Rationale:-}

Local data available from Saudi Arabia are derived from 2 studies conducted in Eastern and central regions. One published in 2000 by Khan et al conducted in Al-Hasa area estimated the prevalence of retinopathy by $13.6 \% .{ }^{11}$ The most recently published study by Aboud FA et al in 2016 is again single center study including Type 2 DM patients attending Al-Wazarat Healthcare Center in the central region. Out of the 748 participants, 111 (14.8\%) had retinopathy, 112 (15\%) had nephropathy, and 5.6\% had neuropathy. Approximately 63 (8.7\%) had myocardial infarction (MI), 49 (6.6\%) had cerebrovascular accident (CVA). The prevalence of both micro- and macro- vascular changes in the same patient were $42(5.6 \%) .{ }^{12}$ Overall, the prevalence of microvascular and macrovascular complications of Diabetes Mellitus seem to be lower than reported in international studies such as the United Kingdom Prospective Diabetes Study (UKPDS). ${ }^{13}$

This situation highlights the need to consistently assess the current prevalence of microvascular and macrovascular diabetic complications in the western region of Saudi Arabia and to put in place some actions in order to improve the quality of care of these patients.

The aim of this study is to explore the prevalence of microvascular and macrovascular complications among male and female patients having diabetes for more than 10 years at King Fahad General Hospital, Jeddah Saudi Arabia. 


\section{Study objectives:-}

Primary:-

To assess the cumulative and individual prevalence of microvascular and macrovascular complications among study population.

\section{Secondary:-}

To Map Cardiovascular Risk Factors among study population.

Correlate diabetic complication prevalence with level of diabetes control (A1C target level)

\section{Study Design:-}

Description of the study design:-

A retrospective cross sectional study design will be used. Patients' files will be used as source of data collection in a brief case report form. To avoid duplication unique patient file number will be used as subject number preceded by investigator number. A random data source verification of $20 \%$ of the case report forms as quality control of collected data.

\section{Duration of study participation:-}

Data will be collected as one single visit. Patients will be enrolled over a period of one month.

\section{Evaluation Criteria:-}

Primary evaluation criteria:-

Number of Micro/Macrovascular complications

Proportion of patients having micro/macro complications

Frequency of micro/macrovascular complications per patients

Proportion of individual micro/macrovascular complication among study population.

\section{Secondary evaluation Criteria:-}

Proportion of Cardiovascular risk factors among study population and its correlation to DM vascular complications. Difference in prevalence of micro/macrovascular and individual complications in patients with target $\mathrm{A} 1 \mathrm{C}<8 \%$ or

$>8 \%$ at the time of diagnosis.

\section{Selection of Patients:-}

\section{Sample Size:-}

It is planned the 3 investigators to recruit 375 patients in King Fahad General Hospital, Jeddah, Saudi Arabia.

\section{Inclusion Criteria:-}

Male or Female patients of all age groups.

Established diagnosis of DM for more than 10 years.

Patients presented to the outpatient clinic during the last three months.

\section{Exclusion Criteria:-}

Incomplete patient file

Modalities of recruitment

To allow extrapolation of the results to the broadest possible population, all consecutive patients who meet the eligibility criteria will enrolled in the study. Each selected investigator will include about 125 consecutive patients' files that meet eligibility criteria. This consecutive recruitment will limit biases of patient selection. The total recruitment period will be 1 month.

\section{Data Collection:-}

\section{Data Collection Schedule:-}

All data will be collected during one single visit (see Section 2.2 Study Flow Chart) in an individual Case Report Form (CRF). 
Data Collected:-

Data will be collected from the medical chart. No tests will be specifically done for this study.

Patient Data:-

The Following Data Will Be Collected:

$>$ Date of last visit,

$>$ Inclusion/exclusion criteria check,

$>$ Patient profile:-

* Demographic characteristics (age, gender, , , marital status, e),

* Lifestyle habits (smoking status, , physical activity),

* Vital signs, weight, waist circumference, body mass index, blood pressure).

* Family History of DM and Cardiovascular disease

* Relevant history of risk factors for DM

$>$ Characteristics of Diabetes:-

* Type of Diabetes

* Duration of diabetes,

Cardiovascular Risk Factors:-

$>$ Microvascular Complications:-

* Retinopathy (Date of Diagnosis, A1C level)

* Nephropathy (Date of Diagnosis, A1C level)

* Neuropathy (Date of Diagnosis, A1C level)

> Macrovascular Complications:-

* Ischemic heart Disease (Type, Date of Diagnosis, A1C level)

* Cerebrovascular Disease (Type, Date of Diagnosis, A1C level)

* Peripheral vascular Disease (Type, Date of Diagnosis, A1C level)

Site/ Investigator questionnaire:-

Not applicable.

Patient/Subject tracking log:-

Not applicable:-

Logistic Aspects:-

Not applicable.

\section{Management of data:-}

Data Collection, Validation and data quality control at mandated CRO LEVEL:-

Data will be collected using paper CRFs. Patient profile and medical history will be collected from clinical records which are considered as source documents.

The computerized handling of the data by the mandated Contract Research Organization (CRO) may generate additional requests to which the participating investigator is obliged to respond by confirming or modifying the data questioned. The requests with their responses will be appended to the CRFs held by the Investigators.

Data collection and validation procedures will be detailed in appropriate operational Documents:Monitoring And Site data quality control:-

Data quality control will be performed at site level, where each investigator will randomly select and review $10 \%$ of CRF filled by his/her colleague against the patient file. That means $20 \%$ of the the CRFs will be cross checked.

Data quality control must be performed by qualified designated personnel. The methodology of data quality control and appropriate consecutive corrective actions will be detailed in the study manual. 


\section{Pharmacovigilance:-}

The investigator should ensure that no product names / brand names are collected through the CRF. For this disease observational study, there will be no systematic collection of safety data.

However, all adverse drug reactions (ADRs) to any product that occurred during the course of the study must be recorded and transmitted to SFDA within 24 hours e.g. ADRs that are discovered at the time of a reviewing patents' files, data source verification and cross checking by another investigator or ADRs discovered during data entry into the database.

According to the International Conference on Harmonization (ICH) E2D guideline, ADRs are noxious and unintended responses to a medicinal product (the phrase 'responses to a medicinal product' means that a causal relationship between a medicinal product and an $\mathrm{AE}$ is at least a reasonable possibility).

According to local regulation, investigators must comply with Saudi Arabian regulations for the spontaneous reporting of ADRs as described in local regulatory requirements issued by Drug Sector/Saudi Food and Drug Authority (SFDA) which relate to Good Pharmacovigilance Practice Guidelines dated 01-SEP-2015, which describe the responsibility of the investigators and sponsor in reporting ADRs to SFDA / National Pharmacovigilance Center.

Per the ICH E2A guideline, a serious ADR is any untoward medical occurrence that at any dose:

$>$ results in death,

$>$ is life threatening (a reaction in which the patient was at risk of death at the time of the reaction e.g. allergic reaction: angioedema of the larynx would be life-threatening),

$>$ requires inpatient hospitalization or results in prolongation of existing hospitalization,

$>$ results in persistent or significant disability / incapacity e.g. loss of visual acuity, fractured neck of femur, convulsions with neurologic sequelae,

$>$ is a congenital anomaly / birth defect, during pregnancy or after birth or any anomaly that results in fetal loss?

$>$ is a medically important reaction (reactions that may not be immediately life-threatening or result in death or hospitalization but may jeopardize the patient or require intervention to prevent one of the other outcomes listed in the definition of seriousness). NB: it is the responsibility of the investigator to assess these events as medically important.

The investigators are responsible within 24 hours from his/her awareness of the ADR with special care to follow up serious cases until final outcome/resolution.

SFDA requires the investigators to report the following special cases:-

$>$ Drug exposure via parent, with or without ADR,

$>$ Lack of efficacy,

$>$ Overdose (intentional or unintentional) with or without ADR,

$>$ Drug misuse, with or without ADR,

$>$ Drug abuse,

$>$ Unintended beneficial effects,

$>$ Medication errors, with or without ADR,

$>$ Suspected transmission of infectious agents.

SFDA ADR form is attached in Annex I.

\section{Safety Observations:-}

$>$ The Investigator should take all appropriate measures to ensure the safety of the patients, notably he/she should follow up the outcome of any ADR related to any product (clinical signs, laboratory values or other, etc.) until the return to normal or consolidation of the patient's condition;

$>$ In case of any serious ADR related to any product, the patient must be followed up until clinical recovery is complete and laboratory results have returned to normal, or until progression has been stabilized. This may imply that follow-up will continue after the patient has left the study;

$>$ According to the American Diabetes Association, hypoglycemia is a condition characterized by abnormally low blood glucose levels, usually falling below $70 \mathrm{mg} / \mathrm{dL}$.

$>$ The Investigator will assess the severity of all AEs/SAEs (including hypoglycemia) as Mild, Moderate, or Severe, based on the following definitions (developed from Clinical Data Interchange Standards Consortium CDISC Study Data Tabulation Model standard terminology v3.1.1): 
- Mild: A type of $\mathrm{AE}$ that is usually transient and may require only minimal treatment or therapeutic intervention. The event does not generally interfere with usual activities of daily living.

- Moderate: A type of AE that is usually alleviated with additional specific therapeutic intervention. The event interferes with usual activities of daily living, causing discomfort, but poses no significant or permanent risk of harm to the research participant.

- Severe: A type of AE that interrupts usual activities of daily living, or significantly affects clinical status, or may require intensive therapeutic intervention.

Statistical Considerations:-

Determination of Sample Size:-

Sample size: 375 patients

Statistical power and sample size justification:-

According to Litwak et al. 2013; the prevalence of microvascular and macrovascular complications among diabetic patients in Middle East/Gulf area including Saudi Arabia was 65.8\% and 28.7\% respectively; accordingly:

Sample size for Microvascular complications: In our study; it is hypothesised that the prevalence of microvascular complications among diabetic patients would be $60 \%$ with a precision of $\pm 5 \%$. A sample size of 369 individuals will allow for a confidence level of $95 \%$ considering a 2 -talied test.

Sample size for Macrovascular complications: In our study; it is hypothesised that the prevalence of macrovascular complications among diabetic patients would be $25 \%$ with a precision of $\pm 5 \%$. A sample size of 288 individuals will allow for a confidence level of $95 \%$ considering a 2-talied test.

Adopting the higher sample size rounded to 375 subject would be required.

Distribution by country or region:-

Limited to one center as pilot study

\section{Disposition of patients:-}

No tracking log will be used in the study. However, all investigators and patients included in the study will be accounted for. The study enrollment period (dates of first and last patients recruited, duration of recruitment period) and the number of patients per investigator will be given.

\section{Analysis Populations:-}

Two populations of patients will be defined:

$>$ The enrolled population: all patients included in the study,

$>$ The analysis population: all patients with DM included in the study who fulfilled eligibility criteria.

\section{Statistical Methods:-}

This section provides specifications for preparation of a final Statistical Analysis Plan (SAP), which will be issued prior to database lock. Any differences compared to this statistical section should be identified and documented in the final SAP. Statistical analysis will be based on all patients enrolled in the registry. Descriptive analysis will be performed. Patients' characteristics (demographic data, risk profile, treatment, etc.) will be described for all the included patients.

Quantitative data will be summarized using mean, median, standard deviation and range for continuous parameters and counts and percentages for categorical parameters. All statistical tests will be performed using two-tailed tests at a 5\% level of significance. Moreover, statistical test will be performed on comparing different groups. Chi square test will be used to compare between categorical parameters. The results will be calculated at $95 \% \mathrm{CI}$ and $5 \%$ level of significance.

For parametric data; two-tailed paired t-test and repeated measures ANOVA will be used when comparing between repeated measurements while independent t-test and one way ANOVA when comparing between study groups. Non-parametric tests of significance would be used with data not normally distributed. Logistic Regression analysis would be performed to detect positive and negative predictors of microvascular and macrovascular complications 
Analyses Variables:-

Information about the patients will be collected through the CRF. All data collected during the registry will be analyzed in the appropriate descriptive analysis. Statistical analysis will be performed by a statistical programmer using SAS software, release 9.4 or higher (SAS institute, Cary, NC, U.S.A).

\section{Interim Analysis:-}

No interim analysis is planned for this registry.

\section{Responsibilities:-}

\section{Study Committees:-}

The chairperson will be responsible for coordinating the conduct of this study. She/He was involved in the preparation and approval of the protocol and its amendment(s), will assess the progress of the study at site level and is given full authority for presentation/publication of the results.

\section{Responsibilities of the Investigators:-}

The Investigator will perform the study in accordance with this protocol, applicable local regulations and international guidelines.

\section{It is the Investigator's responsibility to:-}

$>$ Fill in the CRF and record all data pertinent to the study. She/he will ensure that the information reported in the $\mathrm{CRF}$ is precise and accurate.

The ICF and the Information Sheet used by the Investigator for obtaining the Patient's Informed Consent must be reviewed and approved by the Sponsor prior to submission to the appropriate Ethics Committee (IRB/IEC) for approval / favorable opinion.

The Investigators are responsible for taking all reasonable steps and providing adequate resources to ensure the proper conduct of the study.

Investigators are responsible for:

$>$ Local submission(s) complying with data protection rules,

$>$ Any other local submission(s).

\section{Ethical and Regulatory Standards:-}

\section{Ethical Principles:-}

This study will be conducted in accordance with the principles laid by the $18^{\text {th }}$ World Medical Assembly (Helsinki, 1964) and all subsequent amendments.

\section{Laws and Regulations:-}

This study will be conducted in accordance with the guidelines for Good Epidemiology Practice [US (14) \& European(16)].

The Investigators should locally ensure all necessary regulatory submissions (e.g. IRB/IEC) are performed in accordance with local regulations including local data protection regulations.

\section{Administrative Expectations:- \\ Record Retention:-}

The Investigatorshall arrange for the retention of study documentation until the end of the study. In addition the Investigatorwill comply with specific local regulations/ recommendations with regards to patient record retention.

It is recommended that the Investigator retains the study documents at least 5 years (5) after the completion or discontinuation of the study.

However, applicable regulatory requirements should be taken into account in the event that a longer period is required. 


\section{Confidentiality:-}

All material, information (oral or written) and unpublished documentation provided by the Investigator, including the present protocol and the CRF, are exclusive property of the investigator.

These materials or information (both global and partial) cannot be given or disclosed by the Investigators or by any person of her/his group to unauthorized persons without the prior formal written consent of Investigators.

The Investigatorshall consider as confidential all the information received, acquired or deduced during the study and will take all necessary steps to ensure that there is no break of confidentiality, other than for information to be disclosed by law.

\section{Data protection:-}

The patients' personal data and the Investigators' personal data which may be included in the study database shall be treated in compliance with all local applicable laws and regulations.

When archiving or processing personal data pertaining to the Investigatorand/or to the patients, The investigators and delegated CRO shall take all appropriate measures to safeguard and prevent access to this data by any unauthorized third party.

\section{Insurance:-}

Not applicable.

\section{Premature discontinuation of the study:-}

The investigators can decide at any time and for any reason to prematurely stop or to interrupt the study; the decision will be communicated in writing to If appropriate, according to Saudi regulations, Ethic Committee(s) (IRB/IEC) and SFDA should be informed.

Ownership and use of data and Study Results:-

No use of the data will be possible without the authorisation of the Investigators conducting the study.

\section{Publications:-}

All study Investigators will have full authority of primary presentation and/or primary publication of results. No other publication is allowed before the primary publication. Any subsequent presentation or publication by a study participant (including for substudies) must be approved by the investigators and make reference to the study and the primary publication.

\section{ProtocolAmendments:-}

Any change to the protocol will be recorded in a written amendment, which will be signed by the Investigator. Amendment to the protocol may require regulatory submissions (e.g. IRB/IEC) in accordance with Saudi regulations.

\section{Bibliographic References:-}

1. IDF atlas $7^{\text {th }}$ edition http://www.idf.org/atlasmap/atlasmap. Chapter 1

2. WHO Study Group on Prevention of Diabetes Mellitus, editor. Prevention of diabetes mellitus. Geneva: World Health Organization; 1994.

3. IDF atlas $7^{\text {th }}$ edition http://www.idf.org/atlasmap/atlasmap, Chapter 4, Pages 78-81

4. Irfan M. Diabetes among Saudis a major issue. Published on Arab News (http://arabnews.com) on November $13^{\text {th }}, 2012$.

5. Nataniel Winer MD, James R, Sowers MD. Epidemiology of Diabetes. J Clin Pharmacol, 2004; 44(4):397-405.

6. Dormandy JA, Charbonnel B, Eckland DJ, Erdmann E, Massi-Benedetti M, Moules IK, et al. Secondary prevention of macrovascular events in patients with type 2 diabetes in the PROactive Study (PROspective pioglitAzone Clinical Trial In Macro Vascular Events): a randomized controlled trial. Lancet 2005; 366: 12791289.

7. Aiello LP, Gardner TW, King GL, Blankenship G, Cavallerano JD, Ferris FL, 3rd, et al. Diabetic retinopathy. Diabetes care 1998; 21: 143-156. 
8. Diabetes.co.uk. Diabetes complications. [Updated 2015; Accessed 2015 May 24/]. Available from: http://www.diabetes. co.uk/diabetes-complications/diabetes-complications.html

9. Mottur-Pilson C, Snow V, Bartlett K. Physician Explanations for failing to comply with "best practices". Eff Clin Pract, 2001; 4:207-213.

10. Cabana MD, Rand CS, Powe NR, Wu AW, Wilson MH, Abboud PA C, Rubin HR. Why don't physicians follow clinical practices guidelines? A framework of improvement. JAMA, 1999; 282(15):1458-1465.

11. Khan AR, Wiseberg JA, Lateef ZAA, Khan SA. Prevalence and Determinants of Diabetic Retinopathy in Al Hasa Region of Saudi Arabia: Primary Health Care Centre Based Cross-Sectional Survey, 2007-2009. Middle East Afr J Ophthalmol 2010; 17: 257-263.

12. Alaboud AF, Tourkmani AM, Alharbi TJ,. Alobikan AH, Abdelhay O, Al Batal SM, et al. Microvascular and macrovascular complications of type 2 diabetic mellitus in Central, Kingdom of Saudi Arabia. Saudi Med J 2016; Vol. 37 (12): 1408-1411.

13. Stratton IM, Adler AI, Neil HAW, Matthews DR, Manley SE, Cull CA, et al. Association of glycaemia with macrovascular and microvascular complications of type 2 diabetes s (UKPDS 35): prospective observational study. BMJ 405-412.

14. Litwak L, Goh SY, Hussein Z, Malek R, Prusty V, Khamseh ME. Prevalence of diabetes complications in people with type 2 diabetes mellitus and its association with baseline characteristics in the multinational A1chieve study. Diabetol Metab Syndr. 2013 Oct 24;5(1):57

15. International Society for Pharmocoepidemiology, April 2007. 'Guidelines for Good Pharmacoepidemiology Practices'.

16. Good Epidemiological Practice (GEP) proper conduct in epidemiology research - IEA European Federation (April 2007).

Annex I:- SFDA Adverse drug reaction form

This study is an epidemiological survey and is not intended to collect safety data about medication used in management of diabetes Mellitus. If the investigator discovered Adverse Drug Reactions during review of patient file, he/she will report it to Saudi National Pharmacovigilance Center by online submission using the electronic form in the below link: https://ade.sfda.gov.sa/ADR1.aspx 